\title{
Images in Anesthesia: Transesophageal echocardiogra- phy during Cesarean section in a Marfan's patient with aortic dissection
}
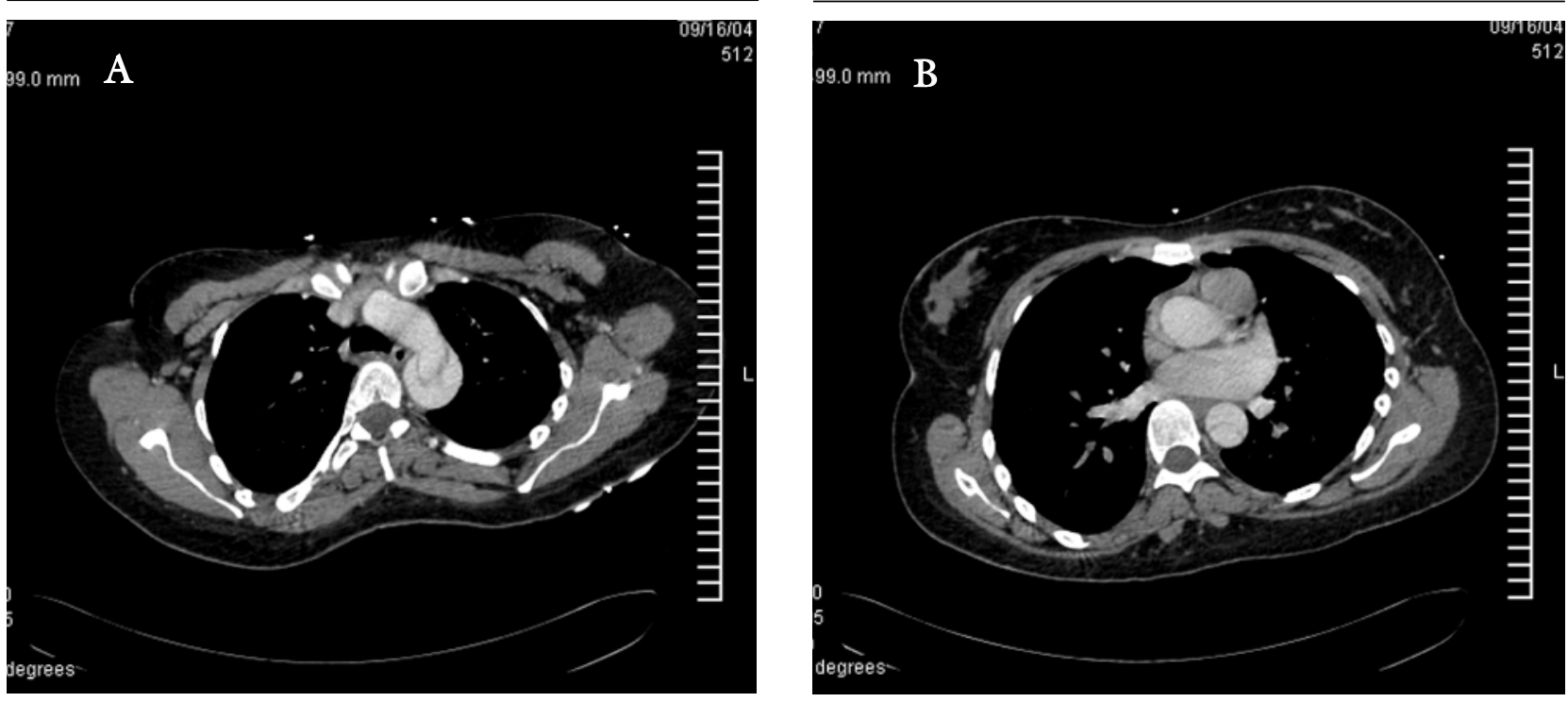

FIGURE A, B Image taken three days before delivery at the level of the thoracic aorta.

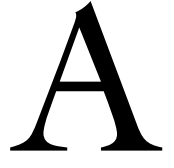

29-yr-old 30-week primigravid woman with Marfan's syndrome presented to the emergency room with chest, back, and epigastric pain. Echocardiography three months earlier had shown mild aortic dilatation (37 $\mathrm{mm}$ ) and mitral valve prolapse. Both transesophageal echocardiography (TEE) and computer tomography confirmed aortic dissection from the take-off of the left subclavian artery that extended past the bifurcation and to the iliac arteries. The patient was hospitalized in the intensive care unit (ICU) near the cardiothoracic surgery department for invasive monitoring that confirmed acute (Stanford) type B aortic dissection. ${ }^{1}$ Antihypertensive, sedative, and steroid therapy were administered immediately. Three days later, because of exacerbation of pain, the patient was scheduled for Cesarean section with preparation for aortic replacement in case of expansion of the dissection into the aortic arch or other unexpected and untoward complications. General anesthesia was induced with fentanyl, propofol, and rocuronium to minimize cardiovascular response to tracheal intuba- tion, and the TEE probe was immediately positioned to monitor the condition of the dissection (video available at www.cja-jca.org). The patient remained hemodynamically stable throughout delivery and was returned to the ICU for continuous conservative treatment. Two weeks later she was discharged in good health. The baby suffered from bilateral hydronephrosis (no genetic information available to date) and mild aortic arch abnormalities compatible with Marfan's syndrome.

In the past, women with Marfan's syndrome were counseled to avoid the risk of pregnancy and delivery. Today, with improved means of identifying patients that facilitate better care, increased longevity, and improved quality of life, more women with Marfan's syndrome are attempting to have babies, despite the high risk of aortic dissection even in the absence of aortic root dilatation prior to pregnancy. For this reason, intraoperative TEE observation ensures an extra measure of real-time control during Cesarean section, particularly in case of acute or chronic aortic dissection, and risk factors such as aortic diameter $\geq 40$ 
$\mathrm{mm},{ }^{2}$ worsening of dilatation, and/or further compromise in cardiac function in the perinatal period. ${ }^{3}$ Potentially, TEE may be recommended for intraoperative real-time control of the aortic state during noncardiovascular surgery in patients with Marfan's syndrome, thereby obviating surgical intervention when not absolutely necessary.

Alexander Ioscovich MD

Deborah Elstein PhD

Shaare Zedek Medical Center, Jerusalem, Israel

E-mail: elstein@szmc.org.il

\section{References}

1 Brar $H B$. Anaesthetic management of a caesarean section in a patient with Marfan's syndrome and aortic dissection. Anaesth Intensive Care 2001; 29: 67-70.

2 Lind J, Wallenburg HC. The Marfan syndrome and pregnancy: a retrospective study in a Dutch population. Eur J Obstet Gynecol Reprod Biol 2001; 98: 28-35.

3 Rabman J, Rabman FZ, Rabman W, al-Suleiman SA, Rabman MS. Obstetric and gynecologic complications in women with Marfan syndrome. J Reprod Med 2003; 48: 723-8. 